V.O. Brzhezytskyi, R.V. Vendychanskyi, Ye.O. Trotsenko, Ya.O. Haran, O.M. Desyatov, V.I. Khominich

\title{
CHARACTERISTICS OF SPECIALIZED SINGLE-PHASE HIGH VOLTAGE DOUBLER RECTIFIER
}

\begin{abstract}
Introduction. To obtain a high voltage direct current, voltage multipliers with a number of cascades of three or more are widely used. At the same time, for voltage levels of $100 \ldots 200 \mathrm{kV}$ there are several advantages of using a specialized single-phase high voltage doubler rectifier. Problem. The main difficulty is that at the moment mathematical modeling has not been worked out for describing modes that use the built-in $R, C$-filter, as well as a nonlinear load in the form of Zener diodes. Goal. Generalization of the results of the authors' previous publications on the development of an analytical method for calculating the modes of a typical high-voltage direct current installation based on a specialized single-phase voltage doubler rectifier. Methodology. Compilation of a system of algebraic linear and nonlinear equations that describe the current and voltage modes in the elements of a typical high-voltage direct current installation with a nonlinear load. Results. It is shown that with the use of linearization of the currentvoltage characteristics of Zener diodes used in the load circuits of a typical high-voltage direct current installation, an analytical solution for the voltages and currents in its elements can be obtained. Originality. The theoretical basis of the complex solution of the system of equations for the currents, voltages and power of the elements of a typical high-voltage direct current installation with the account of nonlinear pulsations is formulated for the first time. Practical value. The obtained theoretical results can be used for calculations, design, optimization of the modes for a wide range of high-voltage direct current installations of technical, technological, and measuring purposes in the range up to $100 . . .200 \mathrm{kV}$. References 16, tables 2, figures 3.

Key words: voltage doubler rectifier, high-voltage Zener diode, current-voltage characteristic.
\end{abstract}

Цель. Обобщение результатов предыдуцих публикаций авторского коллектива по разработке аналитического метода расчёта режимов типовой установки высокого напряжения постоянного тока на основе специализированного однофазного выпрямителя с удвоением напряжения. Методика. Составление системы алгебраических линейных и нелинейных уравнений, описывающих режсиы тока и напряжения в элементах типовой схемы установки высокого напряжения постоянного тока с нелинейной нагрузкой. Результаты. Показано, что с применением линеаризации вольт-амперных характеристик стабилитронов, используемых в цепях нагрузки типовой установки высокого напряжения постоянного тока, может быть получено аналитическое решение для напряжений и токов в её элементах. Научная новизна. Виервые сформулирован теоретический базис комплексного решения системы уравнений для токов, напряжений и мощности элементов типовой установки высокого напряжения постоянного тока с учётом нелинейных пульсаций. Практическая значимость. Полученные теоретические результаты могут быть использованы для расчётов, проектирования, оптимизации режимов иирокого спектра установок высокого напряжения постоянного тока технического, технологического, а также измерительного предназначения в диапазоне до $100 . .200$ кВ. Библ. 16, табл. 2, рис. 3.

Ключевые слова: выпрямитель с удвоением напряжения, высоковольтный стабилитрон, вольт-амперная характеристика.

Introduction. The variety of high voltage applications for steady-state modes of technological equipment (electrostatic precipitators of coal-fired power plants, electro-coloring and coating sputtering devices, electric separators) necessitates the improvement of their power supplies to a level of $100 \ldots 200 \mathrm{kV}$. In connection with this, recently interest in various variants of the improvement of the classical Cockcroft-Walton direct current voltage generator [3] with a number of stages of three or more $[1,2]$ has appeared. At the same time, since high-voltage diodes [4] are of high quality, in order to obtain the above voltage level, it is more efficient to use the Cockroft-Walton generator with only doubling the rectified voltage, and to reduce output voltage ripple - to supplement it with the «built-in» $R, C-$ filter [5].

It should be noted that to date no rigorous mathematical model of the Cockroft-Walton generator has been created. The available publications on this topic give different results on the magnitude of voltage ripples, and there are also no analytical expressions for the shape of pulsing voltage, etc.

In this connection, a new development of the authors' team [6-8] on the creation of elements of the theory of voltages and currents of the Cockroft-Walton generator with voltage doubling, together with the integrated $R, C$ - filter, and, in addition, the possibility of attaching to its load nonlinear elements such as Zener diodes.

The goal of the paper is to generalize the results of previous publications of the authors' group, to formulate and analyze the final analytical expressions for both voltages and currents in the rectifier circuit with voltage doubling in Fig. 1, and for the powers of the elements of the Cockroft-Walton generator with voltage doubling and its integrated $R, C$ - filter, taking into account the nonlinear pulsation modes. To reduce the terminology, we will call such a generator a specialized single-phase high voltage doubler rectifier.

The subject of the research is a specialized highvoltage single-phase rectifier with voltage doubling, its generalized circuit is shown in Fig. 1.

In Fig. 1: $V T$ - high-voltage step-up transformer; $V D_{1}, V D_{2}$ - high-voltage diodes; $C_{1}, C_{2}, C_{3}$ - capacitors; $R_{f}, R_{L V}, r$ - resistors. The branch of $n$ Zener diodes $Z D_{1}, \ldots Z D_{n}$, resistor $r$ and voltmeter $V$ forms the «builtin» high-precision measuring group of the load voltage $U_{L V}$. In this case, the voltage source in the circuit diagram in Fig. 1 is of interest for both technological applications and measuring equipment $[9,10]$. The peculiarity of this voltage source is that by changing the parameters of the elements it is possible to adjust the amplitude and shape

(C) V.O. Brzhezytskyi, R.V. Vendychanskyi, Ye.O. Trotsenko, Ya.O. Haran, O.M. Desyatov, V.I. Khominich 


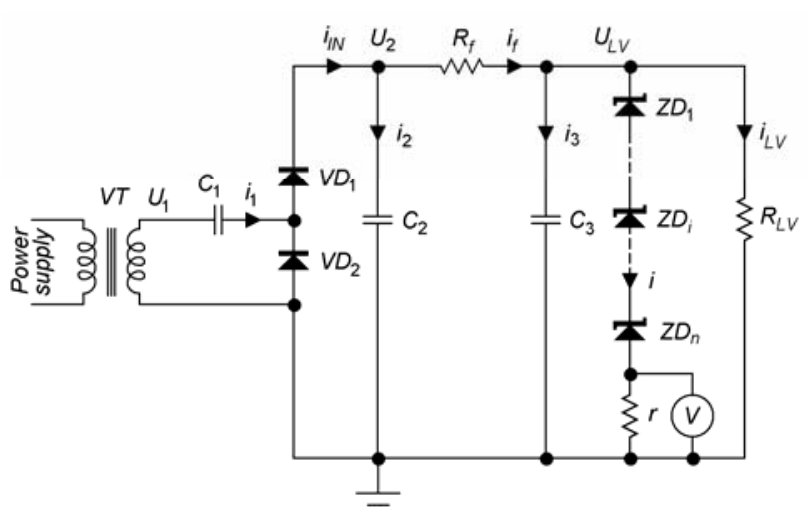

Fig. 1. Functional diagram of rectifier with voltage doubling [5]

of the output voltage ripple $U_{L V}$ in a wide range. The problems of the synthesis of circuits with capacitive energy storage devices, including those using nonlinear electrical loads, are considered in modern publications [11-13]. However, the features of the high-voltage source in Fig. 1 in the well-known publications of other authors have not been studied. The calculated relationships for the voltages and currents of the circuit in Fig. 1 were first obtained in [6-8].

The initial prerequisites for the research are based on a number of conditions:

- a typical current-voltage characteristic of a Zener diode has the form shown in Fig. 2, where $u_{0}, I_{0}$ denote the selected point of its operating mode;

- a differential resistance of a Zener diode $r_{d}=d u_{Z D} / d i_{Z D}$ in its operating domain is far less than the impedance $u_{0} / I_{0}$ (number 1 denotes the linearized currentvoltage characteristic of a Zener diode);

- a capacitance current $C_{Z D} \frac{d u_{Z D}}{d t}$ of a Zener diode is far less than its through-current $I_{0}$ (here $C_{Z D}$ is the interelectrode capacitance of a Zener diode);

- placement of a Zener diode in a metal casing (Fig. 3) completely shields its internal active element from the influence of external electric fields [5].

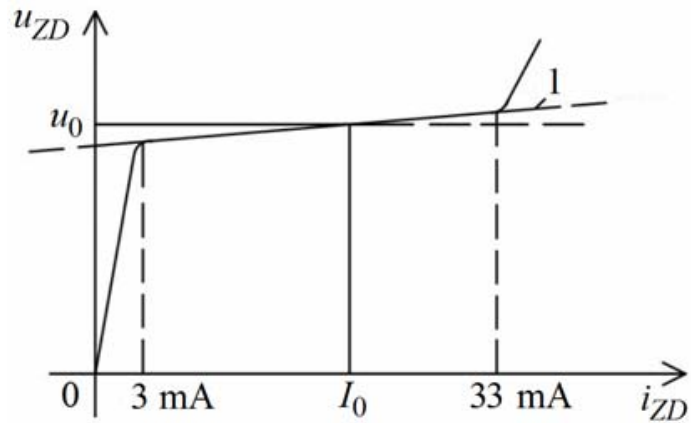

Fig. 2. Current-voltage characteristic of the Zener diode D818D

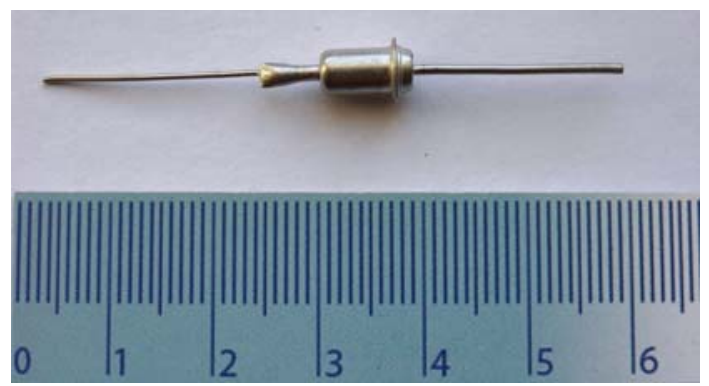

Fig. 3. Photograph of the Zener diode D818D
When these conditions are fulfilled, a series connection of the same type of Zener diodes in the steady state is characterized by the flow of the same current $I_{0}$ through their circuit with the voltage operating point

$$
U_{0}=u_{01}+u_{02}+\ldots+u_{0 i}+\ldots+u_{0 n},
$$

as well as the total differential resistance

$$
R_{d}=r_{d 1}+r_{d 2}+\ldots+r_{d i}+\ldots+r_{d n}
$$

that corresponds to the conclusion of [4] on the admissibility of a serial connection of any number of Zener diodes. The typical «high-voltage» design of insulation of a series of similar Zener diodes eliminates the need to take into account the corona and other phenomena of distributed currents [9].

Derivation of the initial expressions. Then for the instantaneous load voltage $u_{L V}(t)$, one can write:

$$
u_{L V}(t)=U_{0}+I_{0} r+\left(i(t)-I_{0}\right)\left(R_{d}+r\right),
$$

where: $i(t)$ - instantaneous current through a Zener diode and resistor $r$ branch. From here one can get:

$$
i(t)=\frac{u_{L V}(t)-U_{0}-I_{0} r}{R_{d}+r}+I_{0} .
$$

The expressions (1) and (2) are valid within the stabilized domain of the current-voltage characteristic of Zener diodes (Fig. 2) and, therefore, are applicable up to a current ripple level of $\sim 50 \%$ (when $I_{0}$ is selected in the middle part of this domain). The peculiarity of the expressions (1) and (2) is also that they can provide (with an appropriate selection of the parameters $U_{0}, R_{d}$ ) any variants of a series connection of Zener diodes and a resistor $r$, up to the limit: «only the Zener diode» load or «only resistive» load.

Let us write

$$
u_{L V}(t)=U_{0}+I_{0} r+\Delta u(t),
$$

where $\Delta u(t)$ - load voltage ripple as a function of time.

Wherein

$$
\int_{0}^{T} \Delta u(t) d t=0,
$$

where $T=1 / f-$ voltage period of sinusoidal voltage $u_{1}(t)=U_{m} \cdot \sin (\omega t), \omega=2 \pi f-$ angular frequency, $f-$ voltage frequency.

Let us write $i_{3}=C_{3} \cdot d u_{L V}(t) / d t$ (taking into account the losses in the capacitors of the circuit in Fig. 1 slightly refines the results obtained [6]) and $i_{L V}=u_{L V}(t) / R_{L V}$.

Then the current flowing through the resistor $R_{f}$ is:

$$
i_{f}=I_{0}+\frac{\Delta u(t)}{R_{d}+r}+\frac{U_{0}+I_{0} r+\Delta u(t)}{R_{L V}}+C_{3} \frac{d u_{L V}(t)}{d t},
$$

and voltage is:

$$
u_{C 2}(t)=u_{L V}(t)+i_{f} R_{f} .
$$

In its turn, $i_{2}=C_{2} \cdot d u_{C 2}(t) / d t$ and the total current at the input of the right-hand side of the circuit is

$$
\begin{aligned}
& i_{I N}=i_{f}+i_{2}= \\
& =I_{0}+\frac{u_{0}+I_{0} r}{R_{L V}}+\Delta u(t)\left(\frac{1}{R_{L V}}+\frac{1}{R_{d}+r}\right)+ \\
& +\frac{d \Delta u(t)}{d t}\left(C_{3}+C_{2}\left(1+\frac{R_{f}}{R_{L V}}+\frac{R_{f}}{R_{d}+r}\right)\right)+ \\
& +C_{2} C_{3} R_{f} \frac{d^{2} \Delta u(t)}{d t^{2}} .
\end{aligned}
$$


Since the process occurs cyclically, let us assume that at time instant $t_{1}$, the diode $V D_{1}$ «opens», and the current $i_{1}=i_{I N}$ flows (the resistance of the diode $V D_{1}$ in the open state is neglected). At time instant $t_{2}>t_{1}$, the diode $V D_{1}$ «closes» (the resistance of the diode in the closed state is assumed to be infinitely large). In the time interval $t_{2} \leq t \leq T+t_{1}$, the current $i_{I N}=0$. Proceeding from this for a given period of time, from (3) one can obtain an equation for a function of $\Delta u_{1}(t)$ in the form:

$$
\begin{aligned}
& \frac{d^{2} \Delta u_{1}(t)}{d t^{2}}+a_{1} \frac{d \Delta u_{1}(t)}{d t}+a_{2} \Delta u_{1}(t)= \\
& =-\frac{I_{0}+\frac{1}{R_{L V}}\left(U_{0}+I_{0} r\right)}{C_{2} C_{3} R_{f}}
\end{aligned}
$$

where

$$
\begin{gathered}
a_{1}=\frac{C_{3}+C_{2}\left(1+\frac{R_{f}}{R_{L V}}+\frac{R_{f}}{R_{d}+r}\right)}{C_{2} C_{3} R_{f}}, \\
a_{2}=\frac{\frac{1}{R_{L V}}+\frac{1}{R_{d}+r}}{C_{2} C_{3} R_{f}} .
\end{gathered}
$$

A study of the roots $p_{1}, p_{2}$ of the characteristic equation $p^{2}+a_{1} p+a_{2}=0$ shows that its discriminant $D>0$. Thus, one can find the solution for $\Delta u_{1}(t)$ in the form:

$$
\Delta u_{1}(t)=A_{1} e^{p_{1} t}+A_{2} e^{p_{2} t}+\Delta u_{1 s},
$$

where $\Delta u_{1 s}$ - steady-state voltage.

For the industrial power supply frequencies of the circuit in Fig. 1, one can ignore the inductance of its elements. Therefore, in the open state of $V D_{1}$ (during the time interval $t_{1} \leq t \leq t_{2}$ ) one can obtain [6]:

$$
\begin{gathered}
i_{I N}(t)=i_{1}(t), \\
u_{C 2}=U_{m} \sin (\omega t)+U_{m}-\frac{1}{C_{1}} \int_{t_{1}}^{t} i_{1}(t) d t .
\end{gathered}
$$

After differentiating this expression, one can get:

$$
\frac{d u_{C 2}(t)}{d t}=\omega U_{m} \cos (\omega t)-\frac{1}{C_{1}} i_{1}(t)
$$

and, consequently:

$$
i_{1}(t)=C_{1} \omega U_{m} \cos (\omega t)-C_{1} \frac{d u_{C 2}(t)}{d t} .
$$

Using the expression $u_{C 2}(t)=u_{L V}(t)+i_{f}(t) \cdot R_{f}$ and performing its differentiation, and also substituting (6) in (3), one obtain the equation for the function $\Delta u_{2}(t)$ during the time period $t_{1} \leq t \leq t_{2}$ :

$$
\begin{aligned}
& \frac{d^{2} \Delta u_{2}(t)}{d t^{2}}+b_{1} \frac{d \Delta u_{2}(t)}{d t}+b_{2} \Delta u_{2}(t)= \\
& =\frac{C_{1} \omega U_{m} \cos (\omega t)}{R_{f} C_{3}\left(C_{1}+C_{2}\right)}-\frac{I_{0}+\frac{1}{R_{L V}}\left(U_{0}+I_{0} r\right)}{R_{f} C_{3}\left(C_{1}+C_{2}\right)},
\end{aligned}
$$

$$
\begin{gathered}
b_{1}=\frac{C_{3}+\left(C_{1}+C_{2}\right)\left(1+\frac{R_{f}}{R_{L V}}+\frac{R_{f}}{R_{d}+r}\right)}{R_{f} C_{3}\left(C_{1}+C_{2}\right)}, \\
b_{2}=\frac{\frac{1}{R_{L V}}+\frac{1}{R_{d}+r}}{R_{f} C_{3}\left(C_{1}+C_{2}\right)} .
\end{gathered}
$$

The discriminant of the characteristic equation in this case is also greater than zero, and the solution for $\Delta u_{2}(t)$ is found in the form:

$$
\Delta u_{2}(t)=A_{3} \sin (\omega t+\psi)+A_{4} e^{p_{3} t}+A_{5} e^{p_{4} t}+\Delta u_{2 s},(8)
$$

where $\Delta u_{2 s}$ - steady-state voltage, $p_{3}, p_{4}$ - roots of the characteristic equation $p^{2}+b_{1} p+b_{2}=0$. Values of $A_{3}$ and $\psi$ are given by following expressions:

$$
\begin{gathered}
A_{3}=\frac{C_{1} \omega U_{m}}{R_{f} C_{3}\left(C_{1}+C_{2}\right) \sqrt{b_{1}^{2} \omega^{2}+\left(b_{2}-\omega^{2}\right)^{2}}}, \\
\psi=\arctan \left(\frac{b_{2}-\omega^{2}}{b_{1} \omega}\right) .
\end{gathered}
$$

Comparing (5) and (8), one can find:

$$
\Delta u_{1 s}=\Delta u_{2 s}=\Delta u_{s}=-\frac{I_{0}+\frac{1}{R_{L V}}\left(U_{0}+I_{0} r\right)}{\frac{1}{R_{L V}}+\frac{1}{R_{d}+r}},
$$

where $\Delta u_{s}$ - continuous component of the ripple voltage.

Using the invariance of $u_{C 2}$ and $u_{C 3}$ at the time instants $t_{1}, t_{2}$, as well as the determination of $t_{1}$ from the condition $U_{m}\left(1+\sin \left(\omega t_{1}\right)\right)=u_{C 2}\left(t_{1}\right)$, and the time instant $t_{2}$ from the condition $i_{1}\left(t_{2}\right)=0$, and also the expression $\int_{t_{2}}^{T+t_{1}} \Delta u_{1}(t) d t+\int_{t_{1}}^{t_{2}} \Delta u_{2}(t) d t=0$, one can get a system of seven algebraic equations (9) - (15) with seven unknowns: $A_{1}, A_{2}, A_{3}, A_{4}, A_{5}, t_{1}, t_{2}$

$$
\begin{gathered}
\omega A_{3} \cos (\omega \Delta t+\psi)+p_{3} A_{4} e^{p_{3} \Delta t}+p_{4} A_{5} e^{p_{4} \Delta t}= \\
=p_{1} A_{1}+p_{2} A_{2} ; \\
\omega A_{3} \cos (\psi)+p_{3} A_{4}+p_{4} A_{5}= \\
=p_{1} A_{1} e^{p_{1}(T-\Delta t)}+p_{2} A_{2} e^{p_{2}(T-\Delta t)} ; \\
A_{3} \sin (\psi)+A_{4}+A_{5}=A_{1} e^{p_{1}(T-\Delta t)}+A_{2} e^{p_{2}(T-\Delta t)} \\
A_{3} \sin (\omega \Delta t+\psi)+A_{4} e^{p_{3} \Delta t}+A_{5} e^{p_{4} \Delta t}=A_{1}+A_{2} ; \\
\frac{A_{3}}{\omega}(\cos (\psi)-\cos (\omega \Delta t+\psi))=-\Delta u_{s} T- \\
-\frac{A_{1}}{p_{1}}\left(e^{p_{1}(T-\Delta t)}-1\right)-\frac{A_{2}}{p_{2}}\left(e^{p_{2}(T-\Delta t)}-1\right)- \\
-\frac{A_{4}}{p_{3}}\left(e^{p_{3} \Delta t}-1\right)-\frac{A_{5}}{p_{4}}\left(e^{p_{4} \Delta t}-1\right) \\
t_{1}=\frac{1}{\omega} \arcsin \left(\frac{F_{1}}{U_{m}}-1\right)
\end{gathered}
$$

where 


$$
t_{2}=\frac{1}{\omega} \arccos \left(\frac{F_{2}}{\omega U_{m}}\right) .
$$

In (14) $F_{1}$ is given by the expression:

$$
\begin{aligned}
& F_{1}=I_{0} R_{f}+\left(U_{0}+I_{0} r\right)\left(1+\frac{R_{f}}{R_{L V}}\right)+ \\
& +\left(\Delta u_{s}+A_{3} \sin (\psi)+A_{4}+A_{5}\right)\left(1+\frac{R_{f}}{R_{L V}}+\frac{R_{f}}{R_{d}+r}\right)+ \\
& +C_{3} R_{f}\left(\omega A_{3} \cos (\psi)+p_{3} A_{4}+p_{4} A_{5}\right) .
\end{aligned}
$$

In its turn, in (15) $F_{2}$ is given by the expression:

$$
\begin{aligned}
& F_{2}=C_{3} R_{f}\left(-\omega^{2} A_{3} \sin (\omega \Delta t+\psi)+p_{3}^{2} A_{4} e^{p_{3} \Delta t}+\right. \\
& \left.+p_{4}^{2} A_{5} e^{p_{4} \Delta t}\right)+\left(1+\frac{R_{f}}{R_{L V}}+\frac{R_{f}}{R_{d}+r}\right)\left(\omega A_{3} \cos (\omega \Delta t+\psi)+\right. \\
& \left.+p_{3} A_{4} e^{p_{3} \Delta t}+p_{4} A_{5} e^{p_{4} \Delta t}\right) .
\end{aligned}
$$

It should be noted that $\Delta t=t_{2}-t_{1}$, while the relation between $A_{3}$ and $U_{m}$ is defined above.

Equations (9) - (13) are linear with respect to $A_{1}, \ldots$ $A_{j}, \ldots A_{5}$, and therefore the system of equations (9) - (15) can be reduced to three equations with three unknowns $t_{1}$, $t_{2}, A_{j}$. Our experience in calculating (9) - (15) confirms the possibility of obtaining in each particular case a unique solution of the system in a set of real numbers.

Development of theory. The initial expressions (1) - (15) obtained above are derived from the publications of the authors [6-8] and are necessary for the further presentation of the materials in this article.

The advantage of the obtained analytical solution of this problem implies its logical conclusion in the derivation of expressions for the power of the elements of the circuit in Fig. 1 taking into account the voltage and current ripple in these elements (without the assumption of a limitation of their smallness).

The power losses in the $R_{L V}$ load can be found as follows:

$$
P_{L V}=f \int_{t_{2}}^{T+t_{1}} \frac{\left[u_{L V}(t)\right]^{2}}{R_{L V}} d t+f \int_{t_{1}}^{t_{2}} \frac{\left[u_{L V}(t)\right]^{2}}{R_{L V}} d t,
$$

where $u_{L V}(t)=U_{0}+I_{0} r+\Delta u_{1}(t)$ for time interval $t_{2} \leq t \leq T+t_{1}$, and $u_{L V}(t)=U_{0}+I_{0} r+\Delta u_{2}(t)$ for time interval $t_{1} \leq t \leq t_{2}$.

Then let us transform (16) to the following form:

$$
\begin{aligned}
& \frac{R_{L V} P_{L V}}{f}=\left(\frac{R_{L V} P_{L V}}{f}\right)^{(1)}+\left(\frac{R_{L V} P_{L V}}{f}\right)^{(2)}= \\
& =\int_{t_{2}}^{T+t_{1}}\left[V_{0}+\Delta u_{1}(t)\right]^{2} d t+\int_{t_{1}}^{t_{2}}\left[V_{0}+\Delta u_{2}(t)\right]^{2} d t,
\end{aligned}
$$

where $V_{0}=U_{0}+I_{0} r$.

Let us rewrite formula (17), integrating each component and assuming $V_{0}^{(1)}=V_{0}+\Delta u_{s}$, then one can obtain the following expressions, that allow computing the power losses of $P_{L V}$ taking into account the voltage and current ripple on the load $R_{L V}$ :

$$
\begin{aligned}
& \left(\frac{R_{L V} P_{L V}}{f}\right)^{(1)}=\left(V_{0}^{(1)}\right)^{2}\left(T+t_{1}-t_{2}\right)+\frac{A_{1}^{2}}{2 p_{1}} \times \\
& \times\left[e^{2 p_{1}\left(T+t_{1}-t_{2}\right)}-1\right]+\frac{A_{2}^{2}}{2 p_{2}}\left[e^{2 p_{2}\left(T+t_{1}-t_{2}\right)}-1\right]+ \\
& +\frac{2 V_{0}^{(1)} A_{1}}{p_{1}}\left[e^{p_{1}\left(T+t_{1}-t_{2}\right)}-1\right]+\frac{2 V_{0}^{(1)} A_{2}}{p_{2}} \times \\
& \times\left[e^{p_{2}\left(T+t_{1}-t_{2}\right)}-1\right]+\frac{2 A_{1} A_{2}}{p_{1}+p_{2}}\left[e^{\left(p_{1}+p_{2}\right)\left(T+t_{1}-t_{2}\right)}-1\right] ; \\
& \left(\frac{R_{L V} P_{L V}}{f}\right)^{(2)}=\left(V_{0}^{(1)}\right)^{2}\left(t_{2}-t_{1}\right)+A_{3}^{2} \times \\
& \times\left[\frac{t_{2}-t_{1}}{2}+\frac{1}{4 \omega}\left[\sin (2 \psi)-\sin \left(2\left(\omega\left(t_{2}-t_{1}\right)+\psi\right)\right)\right]+\right. \\
& +\frac{A_{4}^{2}}{2 p_{3}}\left[e^{2 p_{3}\left(t_{2}-t_{1}\right)}-1\right]+\frac{A_{5}^{2}}{2 p_{4}}\left[e^{2 p_{4}\left(t_{2}-t_{1}\right)}-1\right]+ \\
& +\frac{2 V_{0}^{(1)} A_{3}}{\omega}\left[\cos (\psi)-\cos \left[\omega\left(t_{2}-t_{1}\right)+\psi\right]\right]+\frac{2 V_{0}^{(1)} A_{4}}{p_{3}} \times \\
& \times\left[e^{p_{3} \cdot\left(t_{2}-t_{1}\right)}-1\right]+\frac{2 V_{0}^{(1)} A_{5}}{p_{4}}\left[e^{p_{4} \cdot\left(t_{2}-t_{1}\right)}-1\right]+ \\
& +\frac{2 A_{3} A_{4}}{\omega\left[\left(\frac{p_{3}}{\omega}\right)^{2}+1\right]}\left[e^{p_{3}\left(t_{2}-t_{1}\right)\left(\frac{p_{3}}{\omega} \sin \left[\omega\left(t_{2}-t_{1}\right)+\psi\right]-\right.}\right. \\
& \left.\left.-\cos \left[\omega\left(t_{2}-t_{1}\right)+\psi\right]\right)-\frac{p_{3}}{\omega} \sin (\psi)+\cos (\psi)\right]+ \\
& +\frac{2 A_{3} A_{5}}{\omega\left[\left(\frac{p_{4}}{\omega}\right)^{2}+1\right]}\left[e^{p_{4}\left(t_{2}-t_{1}\right)} \times\right. \\
& \times\left(\frac{p_{4}}{\omega} \sin \left[\omega\left(t_{2}-t_{1}\right)+\psi\right]-\cos \left[\omega\left(t_{2}-t_{1}\right)+\psi\right]\right)- \\
& \left.-\frac{p_{4}}{\omega} \sin (\psi)+\cos (\psi)\right]+\frac{2 A_{4} A_{5}}{p_{3}+p_{4}}\left[e^{\left(p_{3}+p_{4}\right)\left(t_{2}-t_{1}\right)}-1\right] \text {. }
\end{aligned}
$$

The expression for the power losses in the group of elements $Z D_{1} \ldots Z D_{n}, r$, taking into account the previous consideration, will look like:

$$
\begin{aligned}
& P_{i}=f \int_{t_{2}}^{T+t_{1}}\left[\left[V_{0}+\Delta u_{1}(t)\right] \cdot\left[\frac{\Delta u_{1}(t)}{R_{d}+r}+I_{0}\right]\right] d t+ \\
& +f \int_{t_{1}}^{t_{2}}\left[\left[V_{0}+\Delta u_{2}(t)\right] \cdot\left[\frac{\Delta u_{2}(t)}{R_{d}+r}+I_{0}\right]\right] d t .
\end{aligned}
$$

Next, let us transform expression (20) to the form:

$$
\begin{aligned}
& \frac{P_{i}}{f}=\left(\frac{P_{i}}{f}\right)^{(1)}+\left(\frac{P_{i}}{f}\right)^{(2)}= \\
& =\int_{t_{2}}^{T+t_{1}}\left[V_{0} I_{0}+\Delta u_{1}(t)\left(I_{0}+\frac{V_{0}}{R_{d}+r}\right)+\frac{\left(\Delta u_{1}(t)\right)^{2}}{R_{d}+r}\right] d t+ \\
& +\int_{t_{1}}^{t_{2}}\left[V_{0} I_{0}+\Delta u_{2}(t)\left(I_{0}+\frac{V_{0}}{R_{d}+r}\right)+\frac{\left(\Delta u_{2}(t)\right)^{2}}{R_{d}+r}\right] d t .
\end{aligned}
$$


After integrating each component, the following expressions can be obtained, that allow calculating power losses in the group of elements $Z D_{1} \ldots Z D_{n}, r$ :

$$
\begin{aligned}
& \left(\frac{P_{i}}{f}\right)^{(1)}=V_{0} I_{0}\left(T+t_{1}-t_{2}\right)+\frac{I_{0}^{(1)} A_{1}}{p_{1}}\left[e^{p_{1}\left(T+t_{1}-t_{2}\right)}-1\right]+ \\
& +\frac{I_{0}^{(1)} A_{2}}{p_{2}}\left[e^{p_{2}\left(T+t_{1}-t_{2}\right)}-1\right]+I_{0}^{(1)} \Delta u_{\mathrm{s}}\left(T+t_{1}-t_{2}\right)+ \\
& +\frac{V_{0}^{(2)} A_{1}^{2}}{2 p_{1}}\left[e^{2 p_{1}\left(T+t_{1}-t_{2}\right)}-1\right]+\frac{V_{0}^{(2)} A_{2}^{2}}{2 p_{2}} \times \\
& \times\left[e^{2 p_{2}\left(T+t_{1}-t_{2}\right)}-1\right]+V_{0}^{(2)}\left(\Delta u_{\mathrm{s}}\right)^{2}\left(T+t_{1}-t_{2}\right)+ \\
& +\frac{2 V_{0}^{(2)} A_{1} A_{2}}{p_{1}+p_{2}} \cdot\left[e^{\left(p_{1}+p_{2}\right)\left(T+t_{1}-t_{2}\right)}-1\right]+\frac{2 V_{0}^{(2)} \Delta u_{\mathrm{s}} A_{1}}{p_{1}} \times \\
& \times\left[e^{p_{1}\left(T+t_{1}-t_{2}\right)}-1\right]+\frac{2 V_{0}^{(2)} \Delta u_{s} A_{2}}{p_{2}}\left[e^{p_{2}\left(T+t_{1}-t_{2}\right)}-1\right] ; \\
& \left(\frac{P_{i}}{f}\right)^{(2)}=V_{0} I_{0}\left(t_{2}-t_{1}\right)+\frac{I_{0}^{(1)} A_{3}}{\omega}[\cos (\psi)- \\
& \left.-\cos \left[\omega\left(t_{2}-t_{1}\right)+\psi\right]\right]+\frac{I_{0}^{(1)} A_{4}}{p_{3}}\left[e^{p_{3}\left(t_{2}-t_{1}\right)}-1\right]+ \\
& +\frac{I_{0}^{(1)} A_{5}}{p_{4}}\left[e^{p_{4}\left(t_{2}-t_{1}\right)}-1\right]+I_{0}^{(1)} \Delta u_{s}\left(t_{2}-t_{1}\right)+V_{0}^{(2)} A_{3}^{2} \times \\
& \times\left[\frac{1}{2}\left(t_{2}-t_{1}\right)+\frac{1}{4 \omega}\left[\sin (2 \psi)-\sin \left(2\left(\omega\left(t_{2}-t_{1}\right)+\psi\right)\right)\right]+\right. \\
& +\frac{V_{0}^{(2)} A_{4}^{2}}{2 p_{3}}\left[e^{2 p_{3}\left(t_{2}-t_{1}\right)}-1\right]+\frac{V_{0}^{(2)} A_{5}^{2}}{2 p_{4}}\left[e^{2 p_{4}\left(t_{2}-t_{1}\right)}-1\right]+ \\
& \left.+V_{0}^{(2)}\left(\Delta u_{\mathrm{s}}\right)^{2}\left(t_{2}-t_{1}\right)+\frac{2 V_{0}^{(2)} A_{3} A_{4}}{\omega\left[\left(\frac{p_{3}}{\omega}\right)^{2}+1\right]}\right]^{p_{3}\left(t_{2}-t_{1}\right)} \times \\
& \times\left(\frac{p_{3}}{\omega} \sin \left[\omega\left(t_{2}-t_{1}\right)+\psi\right]-\cos \left[\omega\left(t_{2}-t_{1}\right)+\psi\right]\right)- \\
& \left.-\frac{p_{3}}{\omega} \sin (\psi)+\cos (\psi)\right]+\frac{2 V_{0}^{(2)} A_{3} A_{5}}{\omega\left[\left(\frac{p_{4}}{\omega}\right)^{2}+1\right]}\left[e^{p_{4}\left(t_{2}-t_{1}\right)} \times\right. \\
& \times\left(\frac{p_{4}}{\omega} \sin \left[\omega\left(t_{2}-t_{1}\right)+\psi\right]-\cos \left[\omega\left(t_{2}-t_{1}\right)+\psi\right]\right)- \\
& \left.-\frac{p_{4}}{\omega} \sin (\psi)+\cos (\psi)\right]+\frac{2 V_{0}^{(2)} \Delta u_{S} A_{3}}{\omega}[\cos (\psi)- \\
& \left.-\cos \left[\omega\left(t_{2}-t_{1}\right)+\psi\right]\right]+\frac{2 V_{0}^{(2)} A_{4} A_{5}}{p_{3}+p_{4}}\left[e^{\left(p_{3}+p_{4}\right)\left(t_{2}-t_{1}\right)}-1\right]+ \\
& +\frac{2 V_{0}^{(2)} \Delta u_{\mathrm{s}} A_{4}}{p_{3}} \cdot\left[e^{p_{3}\left(t_{2}-t_{1}\right)}-1\right]+ \\
& +\frac{2 V_{0}^{(2)} \Delta u_{\mathrm{S}} A_{5}}{p_{4}}\left[e^{p_{4}\left(t_{2}-t_{1}\right)}-1\right],
\end{aligned}
$$

where the values of $i_{f 1}, i_{f 2}$ have the following form:

$$
\begin{aligned}
& i_{f 1}=I_{0}+\frac{U_{0}+I_{0} r}{R_{L V}}+\Delta u_{1}(t)\left(\frac{1}{R_{L V}}+\frac{1}{R_{d}+r}\right)+ \\
& +C_{3} \frac{d \Delta u_{1}(t)}{d t} \\
& i_{f 2}=I_{0}+\frac{U_{0}+I_{0} r}{R_{L V}}+\Delta u_{2}(t)\left(\frac{1}{R_{L V}}+\frac{1}{R_{d}+r}\right)+ \\
& +C_{3} \frac{d \Delta u_{2}(t)}{d t} .
\end{aligned}
$$

Then let us transform expression (24) to the form:

$$
\begin{aligned}
& \frac{P_{f}}{R_{f} f}=\left(\frac{P_{f}}{R_{f} f}\right)^{(1)}+\left(\frac{P_{f}}{R_{f} f}\right)^{(2)}= \\
& =\int_{t_{2}}^{T+t_{1}}\left(I_{0}^{(2)}+\Delta u_{1}(t)\left(\frac{1}{R_{L V}}+\frac{1}{R_{d}+r}\right)+C_{3} \frac{d \Delta u_{1}(t)}{d t}\right)^{2} d t+ \\
& +\int_{t_{1}}^{t_{2}}\left(I_{0}^{(2)}+u_{2}(t)\left(\frac{1}{R_{L V}}+\frac{1}{R_{d}+r}\right)+C_{3} \frac{d \Delta u_{2}(t)}{d t}\right)^{2} d t,
\end{aligned}
$$

where $I_{0}^{(2)}=I_{0}+\left(U_{0}+I_{0} r\right) / R_{L V}$.

After integrating each component, one can obtain the following expressions that allow calculating the power losses in the active filter resistance of the installation:

$$
\begin{aligned}
& \left(\frac{P_{3}}{R_{f} f}\right)^{(1)}=\left(I_{0}^{(3)}\right)^{2}\left(T+t_{1}-t_{2}\right)+\frac{X_{4}^{2}}{2 p_{1}}\left[e^{2 p_{1}\left(T+t_{1}-t_{2}\right)}-1\right]+ \\
& +\frac{X_{5}^{2}}{2 p_{2}}\left[e^{2 p_{2}\left(T+t_{1}-t_{2}\right)}-1\right]+\frac{2 I_{0}^{(3)} X_{4}}{p_{1}}\left[e^{p_{1}\left(T+t_{1}-t_{2}\right)}-1\right]+ \\
& +\frac{2 I_{0}^{(3)} X_{5}}{p_{2}}\left[e^{p_{2}\left(T+t_{1}-t_{2}\right)}-1\right]+\frac{2 X_{4} X_{5}}{p_{1}+p_{2}} \times \\
& \times\left(e^{\left(p_{1}+p_{2}\right)\left(T+t_{1}-t_{2}\right)}-1\right] \\
& +\frac{1}{4 \omega}\left[\sin (2 \psi)-\sin 2\left[\omega\left(t_{2}-t_{1}\right)+\psi\right]\right]+\left(A_{3} \omega C_{3}\right)^{2} \times \\
& \times\left[\frac{1}{R_{f} f}\left(t_{2}-t_{1}\right)+\frac{1}{4 \omega}\left[\sin \left(2\left[\omega\left(t_{2}-t_{1}\right)+\psi\right]\right)-\sin (2 \psi)\right]+\right. \\
& +\frac{X_{2}^{2}}{2 p_{3}}\left[e^{2 p_{3}\left(t_{2}-t_{1}\right)}-1\right]+\frac{X_{3}^{2}}{2 p_{4}}\left[e^{2 p_{4}\left(t_{2}-t_{1}\right)}-1\right]+ \\
& +\frac{2 I_{0}^{(3)} X_{1}}{\omega}\left[\cos (\psi)-\cos \left[\omega\left(t_{2}-t_{1}\right)+\psi\right]+\right. \\
& +2 I_{0}^{(3)} A_{3} C_{3}\left[\sin \left[\omega\left(t_{2}-t_{1}\right)+\psi\right]-\sin (\psi)\right]+\frac{2 I_{0}^{(3)} X_{2}}{p_{3}} \times
\end{aligned}
$$




$$
\begin{gathered}
\times\left[e^{p_{3}\left(t_{2}-t_{1}\right)}-1\right]+\frac{2 I_{0}^{(3)} X_{3}}{p_{4}}\left[e^{p_{4}\left(t_{2}-t_{1}\right)}-1\right]+\frac{X_{1} A_{3} C_{3}}{2} \times \\
\times\left[\cos (2 \psi)-\cos \left(2\left[\omega\left(t_{2}-t_{1}\right)+\psi\right]\right)\right]+ \\
+\frac{2 X_{1} X_{2}}{\omega\left[\left(\frac{p_{3}}{\omega}\right)^{2}+1\right]}\left[e^{p_{3}\left(t_{2}-t_{1}\right)} \times\right. \\
\times\left(\frac{p_{3}}{\omega} \sin \left[\omega\left(t_{2}-t_{1}\right)+\psi\right]-\cos \left[\omega\left(t_{2}-t_{1}\right)+\psi\right]\right)- \\
\left.-\frac{p_{3}}{\omega} \sin (\psi)+\cos (\psi)\right]+\frac{2 X_{1} X_{3}}{\left[\left(\frac{p_{4}}{\omega}\right)^{2}+1\right]}\left[e^{p_{4}\left(t_{2}-t_{1}\right)} \times\right.
\end{gathered}
$$$$
\times\left(\frac{p_{4}}{\omega} \cdot \sin \left[\omega \cdot\left(t_{2}-t_{1}\right)+\psi\right]-\cos \left[\omega \cdot\left(t_{2}-t_{1}\right)+\psi\right]\right)-
$$$$
\left.\frac{p_{4}}{\omega} \cdot \sin \psi+\cos \psi\right]+\frac{2 X_{2} A_{3} C_{3}}{\left(\frac{p_{3}}{\omega}\right)^{2}+1}\left[e^{p_{3} \cdot\left(t_{2}-t_{1}\right)} \times\right.
$$$$
\times\left(\frac{p_{3}}{\omega} \cdot \cos \left[\omega \cdot\left(t_{2}-t_{1}\right)+\psi\right]+\sin \left[\omega \cdot\left(t_{2}-t_{1}\right)+\psi\right]\right)-
$$$$
\left.-\frac{p_{3}}{\omega} \cos (\psi)-\sin (\psi)\right]+\frac{2 X_{3} A_{3} C_{3}}{\left(\frac{p_{4}}{\omega}\right)^{2}+1}\left[e^{p_{4}\left(t_{2}-t_{1}\right)} \times\right.
$$$$
\times\left(\frac{p_{4}}{\omega} \cos \left[\omega\left(t_{2}-t_{1}\right)+\psi\right]+\sin \left[\omega\left(t_{2}-t_{1}\right)+\psi\right]\right)-
$$$$
\begin{gathered}
\left.-\frac{p_{4}}{\omega} \cos (\psi)-\sin (\psi)\right]+\frac{2 X_{2} X_{3}}{p_{3}+p_{4}} \times \\
\times\left[e^{\left(p_{3}+p_{4}\right)\left(t_{2}-t_{1}\right)}-1\right]
\end{gathered}
$$

where

$$
\begin{gathered}
I_{0}^{(3)}=I_{0}^{(2)}+\Delta u_{\mathrm{s}}\left(\frac{1}{R_{L V}}+\frac{1}{R_{d}+r}\right) ; \\
X_{1}=A_{3}\left(\frac{1}{R_{L V}}+\frac{1}{R_{d}+r}\right) ; \\
X_{2}=A_{4}\left(p_{3} C_{3}+\frac{1}{R_{L V}}+\frac{1}{R_{d}+r}\right) ; \\
X_{3}=A_{5}\left(p_{4} C_{3}+\frac{1}{R_{L V}}+\frac{1}{R_{d}+r}\right) ; \\
X_{4}=A_{1}\left(p_{1} C_{3}+\frac{1}{R_{L V}}+\frac{1}{R_{d}+r}\right) ; \\
X_{5}=A_{2}\left(p_{2} C_{3}+\frac{1}{R_{L V}}+\frac{1}{R_{d}+r}\right) .
\end{gathered}
$$

Approbation of the obtained theoretical results was performed using the calculations of the parameters of the standard installation DETU 08-04-99, that is used in the State verification scheme for means of measuring the direct current electric voltage in the range $1 \ldots 180 \mathrm{kV}$ [14].

To the high voltage direct current power supply circuit shown in Fig. 1 were assigned the following parameters that correspond to the installation of DETU 08-04-99 in the modes of rated voltages $V_{0}$ from 1 to $180 \mathrm{kV}: C_{1}$ - charging capacitor $(0.1 \mu \mathrm{F}) ; C_{2}, C_{3}$ - filter capacitors $(0.072 \mu \mathrm{F})$; $R_{f}$ - filter resistance $(1.78 \mathrm{M} \Omega) ; Z D_{1}, \ldots Z D_{i}, \ldots Z D_{n}$ are

\begin{tabular}{|c|c|c|c|c|c|}
\hline \multicolumn{6}{|c|}{$\begin{array}{l}\text { Calculation results for } 27 \text { power modes } \\
\text { of the DETU 08-04-99 installation }\end{array}$} \\
\hline$V_{0}, \mathrm{kV}$ & $U_{m}, \mathrm{kV}$ & $\Delta_{1}, \mathrm{~V}$ & $\Delta_{2}, \mathrm{~V}$ & $\Delta_{p}, \%$ & $I_{0}+I_{L V}, \mathrm{~mA}$ \\
\hline 1 & 5.97 & 3.54 & -4.08 & 0.381 & 5.083 \\
\hline 2 & 6.61 & 3.81 & -4.88 & 0.217 & 5.167 \\
\hline 3 & 7.25 & 4.45 & -5.69 & 0.169 & 5.250 \\
\hline 4 & 7.89 & 5.04 & -6.49 & 0.144 & 5.333 \\
\hline 5 & 8.52 & 5.57 & $\begin{array}{l}-7.27 \\
\end{array}$ & 0.128 & 5.417 \\
\hline 6 & 9.16 & 6.04 & -8.04 & 0.117 & 5.500 \\
\hline 7 & 9.80 & 6.44 & -8.81 & 0.109 & 5.583 \\
\hline 8 & 10.44 & 6.78 & -9.54 & 0.102 & 5.667 \\
\hline 9 & 11.08 & 7.07 & -10.26 & 0.096 & 5.750 \\
\hline 10 & 11.72 & 7.31 & -10.97 & 0.091 & 5.833 \\
\hline 20 & 18.27 & 11.41 & -20.65 & 0.080 & 6.667 \\
\hline 30 & 24.66 & 13.09 & -24.15 & 0.062 & 7.500 \\
\hline 40 & 29.27 & 11.71 & -22.07 & 0.042 & 6.667 \\
\hline 50 & 35.22 & 12.46 & -23.82 & 0.036 & 7.083 \\
\hline 60 & 41.17 & 13.18 & -25.51 & 0.032 & 7.500 \\
\hline 70 & 46.09 & 12.18 & -23.86 & 0.025 & 6.944 \\
\hline 80 & 51.90 & 12.63 & -24.97 & 0.023 & 7.222 \\
\hline 90 & 57.71 & 13.09 & -26.05 & 0.021 & 7.500 \\
\hline 100 & 62.04 & 11.11 & -22.32 & 0.017 & 6.389 \\
\hline 110 & 67.70 & 11.39 & -22.88 & 0.016 & 6.528 \\
\hline 120 & 73.36 & 11.67 & -23.43 & 0.015 & 6.667 \\
\hline 130 & 79.02 & 11.95 & -23.97 & 0.014 & 6.806 \\
\hline 140 & 84.68 & 12.22 & -24.51 & 0.013 & 6.944 \\
\hline 150 & 90.34 & 12.49 & -25.03 & 0.012 & 7.083 \\
\hline 160 & 96.00 & 12.76 & -25.55 & 0.012 & 7.222 \\
\hline 170 & 101.66 & 13.02 & -26.07 & 0.012 & 7.361 \\
\hline 180 & 107.32 & 13.29 & -26.59 & 0.011 & 7.500 \\
\hline
\end{tabular}
Zener diodes of the D818D type; $R_{L V}$ is the resistance of the resistive voltage divider.

For the D818D Zener diodes, the value of stabilized current $I_{0}=5 \mathrm{~mA}$ was selected for 27 different values of the rated voltages $V_{0}$ on the load, according to Table 1 . The voltage divider has four values of input rated voltages $V_{0}: 180 \mathrm{kV} ; 90 \mathrm{kV} ; 60 \mathrm{kV} ; 30 \mathrm{kV}$ for which the current of the divider voltage is $I_{L V}=2.5 \mathrm{~mA}$. For the other 23 input voltages of the voltage divider $V_{0}$, its current decreases in proportion to the input voltage.

Calculations were performed to solve the system of equations (9) - (15) for the parameters $r=10 \mathrm{k} \Omega$ for the modes $V_{0}=1 \ldots 10 \mathrm{kV}$ and $r=60 \mathrm{k} \Omega$ for the $V_{0}=20 \ldots 180 \mathrm{kV}$ modes. The value of $r_{d}$ was assumed according to [4] equal to $22 \Omega$ for each Zener diode, and $R_{d}=n r_{d}$, where $n$ is the number of Zener diodes corresponding to each mode $V_{0}$. This quantity is determined based on the average value of the D818D stabilization voltage $u_{0}=9 \mathrm{~V}$.

Based on the calculation results, the maximum positive pulsation values $\Delta u(t)=\Delta_{1}$ and minimum negative pulsation values $\Delta u(t)=\Delta_{2}$ were determined, as well as the pulsation amplitude coefficient:

$$
\Delta_{p}=\frac{\Delta_{1}-\Delta_{2}}{2 V_{0}} 100, \%
$$

The obtained results of calculations are shown in Table 1. Table 1 also shows the value of the total average 
load current $I_{0}+I_{L V}$ (mA) for each DETU 08-04-99 installation operating mode.

From the data in Table 1 it follows that with increase in load voltage $V_{0}$, the pulsation amplitude coefficient $\Delta_{p}$ decreases accordingly. In the $V_{0}=1 \mathrm{kV}$ mode, the pulsation amplitude coefficient $\Delta_{p}=0.381 \%$, and in the $V_{0}=180 \mathrm{kV}$ mode the pulsation amplitude coefficient is $\Delta_{p}=0.011 \%$. The above values of ripple in different operating modes of the installation differ by a factor of 35 .

Using the values of $P_{L V}, P_{i}, P_{f}$ obtained above, one can determine the energy efficiency coefficient of the DETU 08-04-99 installation with nonlinear load:

$$
E F F=\frac{P_{L V}+P_{i}}{P_{L V}+P_{i}+P_{f}} 100=\frac{1}{1+P_{f} /\left(P_{L V}+P_{i}\right)} 100, \% .
$$

Table 2 shows the calculated values of $E F F$ (28) for the DETU 08-04-99 installation for $V_{0}$ modes from 1 to $30 \mathrm{kV}$. For the $V_{0}=180 \mathrm{kV}$ mode, the $E F F$ value is $93.1 \%$.

The calculated results for the DETU 08-04-99 installation, given in Table 1 and Table 2 are confirmed by the data of installation experimental study.

Table 2

The values of the energy efficiency coefficient of the DETU 08-04-99 installation for the $V_{0}$ values from $1 \mathrm{kV}$ to $30 \mathrm{kV}$

\begin{tabular}{|c|c|c|c|c|}
\hline$V_{0}, \mathrm{kV}$ & $P_{L V}, \mathrm{~W}$ & $P_{i}, \mathrm{~W}$ & $P_{f}, \mathrm{~W}$ & $E F F, \%$ \\
\hline 1.0 & 0.09 & 5.25 & 46.15 & 10.38 \\
\hline 2.0 & 0.35 & 10.25 & 47.68 & 18.19 \\
\hline 3.0 & 0.78 & 15.25 & 49.20 & 24.57 \\
\hline 4.0 & 1.37 & 20.25 & 50.77 & 29.86 \\
\hline 5.0 & 2.13 & 25.25 & 52.37 & 34.33 \\
\hline 6.0 & 3.05 & 30.25 & 53.99 & 38.15 \\
\hline 7.0 & 4.14 & 35.25 & 55.63 & 41.45 \\
\hline 8.0 & 5.40 & 40.25 & 57.30 & 44.34 \\
\hline 9.0 & 6.83 & 45.25 & 59.00 & 46.88 \\
\hline 10.0 & 8.42 & 50.25 & 60.72 & 49.14 \\
\hline 20.0 & 34.34 & 101.50 & 79.85 & 62.98 \\
\hline 30.0 & 76.51 & 151.50 & 100.97 & 69.31 \\
\hline
\end{tabular}

The discussion of the results. When determining the energy efficiency coefficient of the high-voltage installation, let us consider the power $P_{\text {out }}=P_{L V}+P_{i}$ as a net power, while the power $P_{f}$ represents the additional power losses in the filter resistance. At the same time, an increase in the resistance of the filter $R_{f}$ is a means of decreasing the amplitude of the voltage ripple in the installation load when increasing voltage $U_{m}$ at the input of the circuit [5].

The peculiarity of developing a mathematical model for the typical high-voltage installation modes (Fig. 1) is that it determines the necessary parameters of the installation in the opposite direction - given average voltage drop across the group of Zener diodes $U_{0}$ for a given average current $I_{0}$ through it. The proposed solution sets the numerical value of the time instant $t_{1}$ - the start of the charging of the installation capacitor $C_{2}$ and the time instant $t_{2}$ - the «disconnection» of the right part of the installation from the capacitance $C_{1}$, and also determines the parameters $A_{1}, A_{2}, A_{3}, A_{4}, A_{5}, p_{1}, p_{2}, p_{3}, p_{4}, \psi, \Delta u_{s}, U_{m}$ depending on the values of $U_{0}, I_{0}, C_{1}, C_{2}, C_{3}, R_{L V}, T, r, R_{d}$, $R_{f}$ by the analytical method, and is new.

The use of Zener diodes in the measuring group of a high-voltage direct current installation allows significantly reducing the pulsation amplitude (up to 3 and more times) and improve the voltage quality on the load [8].
It should also be noted that nowadays professional and demonstration versions of various circuit simulation programs are widely used to simulate voltage multiplication schemes, as well as processes in electrical equipment insulation. To simulate, for example, the phenomenon of partial discharges in the insulation of highvoltage equipment, demonstration versions of the programs are sufficient enough $[15,16]$. However, the number of Zener diodes in the operating DETU 08-04-99 installation is tens of thousands of pieces. In this regard, on the one hand, a complete simulation of such a scheme requires expensive professional circuit simulation programs. On the other hand, as shown in this article, there is no need for a detailed circuit simulation of such a complex scheme. Taking these factors into account, the group of authors made a choice in favor of a generalized analytical solution of the problem posed in this article.

\section{Conclusion.}

1. An analytical method for solving equations for a complex system of a typical high voltage direct current installation based on a voltage doubler rectifier with an integrated $R, C$ - filter, and a measuring group has been developed.

2. The solution obtained is generalized for the case of insertion of Zener diodes into the measuring group, while it is valid within the linearized domain of their currentvoltage characteristic.

3. The use of Zener diodes in the load circuits of the installation makes it possible to significantly reduce the amplitude of voltage ripple, and also to improve the quality of the output voltage of direct current voltage multiplier.

4. A theoretical basis is developed not only for voltages and currents, but also for the electrical power of the elements of a typical high-voltage direct current installation with allowance for nonlinear voltage pulsations.

\section{REFERENCES}

1. Dwivedi C.K., Daigavane M.B. Multi-purpose low cost DC high voltage generator (60 kV output), using Cockcroft-Walton voltage multiplier circuit. International Journal of Science and Technology Education Research, 2011, vol.2(7), pp. 109-119.

2. Cortez D.F., Barbi I. A Family of High Voltage Gain SinglePhase Hybrid Switched-Capacitor PFC Rectifiers. IEEE Transactions On Power Electronics, 2015, vol.30, no.8, pp. 4189-4198. doi: 10.1109/TPEL.2014.2360173.

3. Cockroft J.D., Walton E.T.S. Experiments with high velocity positive ions. (I) Further developments in the method of obtaining high velocity positive ions. Proceedings of the Royal Society A. Mathematical, physical and engineering sciences, 1932, vol.136, no.830, pp. 619-630. doi: 10.1098/rspa.1932.0107.

4. Golomedov A.V. Poluprovodnikovye pribory. Diody vypriamitel'nye, stabilitrony, tiristory: spravochnik [Semiconductors. Rectifier diodes, Zener diodes, thyristors. Directory]. Moscow, Radio and communication Publ., 1988. 347-350. (Rus).

5. Brzhezytskyi V.O., Vendychanskyi R.V., Desiatov O.M., Haran I.A. Rationale diodes and modes of supply of standard units of high voltage direct current. Science news of NTUU «KPI», 2014, no.1, pp. 7-13. (Ukr).

6. Brzhezytskyi V., Desiatov O., Suleimanov V., Khominich V. Analysis of high-voltage cascade generator pulsations of a direct current. Technology audit and production reserves, 2015, vol.1, no.1(21), pp. 56-61. doi:10.15587/2312-8372.2015.37219. (Ukr). 
7. Brzhezitsky V., Desiatov O., Maslychenko I., Anokhin Y. Analytical research mode the voltage of high-voltage cascade generator with nonlinear loads. Scientific Works of National University of Food Technologies, 2015, vol.21, no.3, pp. 183-191. (Ukr).

8. Brzhezitsky V., Desyatov O., Garan J., Babicheva A. Analysis of the current ripple of cascade generator high DC voltage. Scientific Herald of NULES of Ukraine. Series: Technique and energy of APK, 2015, no.209-2, pp. 30-38. (Ukr).

9. Merev A., Hällström J.K. A Reference System for Measuring High-DC Voltage Based on Voltage References. IEEE Transactions On Instrumentation And Measurement, 2015, vol.64, no.1, pp. 184-189. doi: 10.1109/TIM.2014.2338673.

10. Anokhin Y.L., Brzhezytskyi V.O., Haran Ya.O., Masliuchenko I.M., Protsenko O.P., Trotsenko Ye.O Application of high voltage dividers for power quality indices measurement. Electrical engineering \& electromechanics, 2017, no.6, pp. 53-59. doi: 10.20998/2074-272X.2017.6.08.

11. Kravchenko V.I., Petkov A.A. Parametrical synthesis of highvoltage pulse test devices with capacitive energy storage. Electrical engineering \& electromechanics, 2007. no.6, pp. 70-75. (Rus).

12. Shcherba A.A., Suprunovska N.I., Ivashchenko D.S. Modeling of nonlinear resistance of electro-spark load for synthesis of discharge circuit of capacitor by time parameters. Technical electrodynamics, 2014, no.3, pp. 12-18. (Rus).

13. Suprunovska N.I., Shcherba A.A. Processes of energy redistribution between parallel connected capacitors. Technical electrodynamics, 2015, no.4, pp. 3-11. (Rus).

14. DSTU 3863-99 Derzhavnyi standart Ukrainy. Metrolohiia. Derzhavna povirochna skhema dlia zasobiv vymiriuvan elektrychnoi napruhy postiinoho strumu $v$ diapazoni vid 1 do $800 \mathrm{kV}$ [DSTU 3863-99. State standard of Ukraine. Metrology. State verification schedule for means measuring of direct current electric voltage in the range from 1 to $800 \mathrm{kV}$ ]. Kyiv, Derzhstandart of Ukraine Publ., 1999. 10p. (Ukr).
15. Trotsenko Ye., Brzhezitsky V., Protsenko O., Chumack V., Haran Ya. Simulation of partial discharges under influence of impulse voltage. Technology audit and production reserves, 2018, vol.1, no.1(39), pp. 36-41. doi: 10.15587/2312-8372.2018.123309.

16. Trotsenko Ye., Brzhezitsky V., Protsenko O., Chumack V., Haran Ya. Effect of voltage harmonics on pulse repetition rate of partial discharges. Technology audit and production reserves, 2018, vol.2, no.1(40), pp. 37-44. doi: 10.15587/23128372.2018.126626.

Received 30.08.2018

V.O. Brzhezytskyi ${ }^{1}$, Doctor of Technical Science, Professor,

R.V. Vendychanskyi ${ }^{2}$, Deputy Chief of the Research Department of Measurements of Electrical Values,

Ye.O. Trotsenko ${ }^{1}$, Candidate of Technical Science, Associate

Professor,

Ya.O. Haran ${ }^{1}$, Assistant,

O.M. Desyatov ${ }^{1}$, Engineer,

V.I. Khominich ${ }^{1}$, Candidate of Technical Science, Senior

Researcher,

${ }^{1}$ National Technical University of Ukraine «Igor Sikorsky Kyiv Polytechnic Institute»,

37, Prosp. Peremohy, Kyiv, 03056, Ukraine, phone +380442367989 ,

e-mail: v.brzhezitskiy@ukr.net

${ }^{2}$ State Enterprise «All-Ukrainian State Research and Production Center For Standardization, Metrology, Certification and

Consumers Rights Protection» (SE «Ukrmetrteststandard»), 4, Metrolohichna Str., Kyiv, 03168,Ukraine, phone +380445263485

e-mail: hivolt@ukrcsm.kiev.ua 